\title{
Energy Efficient Trust Node Based Routing Protocol (EETRP) to Maximize the Lifetime of Wireless Sensor Networks in Plateaus
}

\author{
https://doi.org/10.3991/ijoe.v15i06.10340 \\ Nandoori Srikanth ${ }^{\bowtie}$, Muktyala Sivaganga Prasad \\ Koneru Lakshmiah Educational Foundation, Green fields, Vaddeswaram, India \\ srilovesnature@gmail.com
}

\begin{abstract}
Wireless sensor networks (WSNs) can extant the individual profits and suppleness with regard to low-power and economical quick deployment for numerous applications. WSNs are widely utilized in medical health care, environmental monitoring, emergencies and remote control areas. Introducing of mobile nodes in clusters is a traditional approach, to assemble the data from sensor nodes and forward to the base station. Energy efficiency and lifetime improvements are key research areas from past few decades. In this research, to solve the energy limitation to upsurge the network lifetime, energy efficient trust node based routing protocol is proposed. An experimental validation of framework is focused on packet delivery ratio, network lifetime, throughput, energy consumption and network loss among all other challenges. This protocol assigns some high energy nodes as trusted nodes, and it decides the mobility of data collector. The energy of mobile nodes, and sensor nodes can save up to a great extent by collecting data from trusted nodes based on their trustworthiness and energy efficiency. The simulation outcome of our evaluation shows an improvement in all these parameters than existing clustering and routing algorithms.
\end{abstract}

Keywords-WSN, EETRP, Mobile Node, Trust Node, Sub-Cluster (SC)

\section{Introduction}

The main objective of wireless sensor network (WSN) is to observe physical or ecological conditions, such as sound, temperature, pressure, vibration, motion or contaminants and to co-operatively pass their data by the network to a central position (i.e., base station or sink) [1]. WSNs are developing as an important and popular ways of provided that prevalent computing environments for several applications and harshly challenged by limited energy. It's essential to use available energy in efficient manner to maintain sustainability in networks [2]. In WSNs, the deployment of sensor nodes (SN) is performed whichever in the arbitrary manner. Firstly, WSNs was similar in nature, i.e. all the $\mathrm{SN}$ and cluster-heads are undistinguishable with reference to power consumption, storage capacity and computing capability [3]. WSN is an arrangement of several nodes for efficient performance of WSN; we have required 
competent routing protocol with well-planned data aggregation methods as well as very low energy consumption. These networks are drastically resource limited by their bound power supply. To figure out this issue hierarchical routing protocol based on clustering is taken into consideration [4].

Mahajan et al., (2014) [5] reported a rank based metric approach which is recommended to choose clusters from the set of sensors in view of network performance parameters that allocates load consistently in the cluster and consume least energy. Heterogeneous mobile sensor network environment wireless sensor network is a dispersed network, which includes a set of independent and self-organized sensor nodes and one or more base station (BS). This sort of network can be designed and arranged to observe and track applications for instance smart environments like smart cities, smart grid, smart home; habitat monitoring and surveillance etc. [6]. In wireless sensor networks, trust model stipulates a significant role in recognizing misbehavior nodes and provided that co-operation amid trustworthy nodes. Trust node plays a most important role to sense a node which is not performing as expected (whichever defective or maliciously). Trust judges the quality of node and their services. It improves the lifetime of networks that motivate anticipations amid future interactions in WSNs [7].

\subsection{Motivation of the paper}

Clustering is a technique which is utilized to progress the lifetime of nodes and several clustering algorithms have been established to enhance the energy balance of the WSN's due to energy is the principal feature of WSN's during data transmission. These algorithms are mainly used for increasing the lifetime of sensor networks. The whole sensing field is distributed into sub clusters. In every sub cluster, few mobile nodes are introduced for various parameters, which include energy or distance from the base station. Introduced a mobile node $(\mathrm{MN})$ in a sub cluster is the initial motivation of this paper, because deployment of $\mathrm{SN}$ and collection of data in plateaus and military areas is a major challenging issue, thus mobile node has to ingest more energy to collect the data from those nodes. Energy efficient trust node based routing protocol was proposed to upsurge the lifetime of the network.

\subsection{Contributions of the paper}

In this research, an Energy efficient trust node based routing protocol (EETRP) was proposed in WSN. Nodes are structured into clusters, cluster is divided into the sub clusters, and we assign some sub-cluster nodes as trust nodes. However, EETRP algorithm is initially performed on all sub cluster nodes to form balanced sensor nodes, proper mobile node and trust node are designated. The main contributions of this research are as follows:

- Energy efficient trust node based routing protocol (EETRP) is proposed, which aims to minimize energy consumption for data transmission in WSN. 
- EETRP protocol is utilized to optimize the sub clustering algorithm rules to upsurge the network lifetime, based on the applications like plateaus and military areas.

- This protocol differs from the traditional clustering approaches due to uneven deployment and placement of sensor nodes in plateaus.

- EETRP utility system to concurrently ponder two elements: trustworthiness of nodes and energy efficiency.

\section{Related Work}

WSNs can extant the individual profits and suppleness as regards low-power and economical fast deployment for a number of applications. Sensor arrangement is an acute problem for some important objectives in WSNs like coverage, lifetime and connectivity. Deployment of tactic was depends on potential field theory to deploy the mobile sensor nodes in an unfamiliar environment to enhance the network coverage [8]. Numerous energy efficient schemes are well-defined as a cluster based routing is found to be a more energy efficient and ascendable way to form sensor nodes [9]. As shortest path spanning trees instinctively have short delay, it is extremely significant to find an energy-efficient shortest path tree for time-critical applications [10]. Hybrid energy efficient distributed clustering (HEED) was reported which is a multi-hop clustering algorithm for WSN. HEED contain distributing energy consumption to prolong network lifetime, reducing energy consumption during $\mathrm{CH}$ selection phase and reduce the control overhead of network [11].

Efficient sleep awake aware (EESAA) is an intelligent routing protocol for WSNs which presented a method of pairing amid nodes. Amid these pairs just a single member node awakes in a every single round to forward sensed data even though the other member node stays in sleep mode to save the energy resources [12]. Deployment of relay nodes results fault-tolerance in heterogeneous WSN with higher network connectivity. Heterogeneous WSN contain sensor nodes with unlike transmission radius. Further relay node proved more advantages when deployed in clustered sensor networks [13]. Chuanhe et al., (2007) [14] was introduced a reputation-based trust management scheme using a stimulus mechanism. Trust management scheme encourages packet forwarding and discourages selfish behaviors based on quantified objective measures and reputation propagation by a one-way hash chain based authentication. The performance of this scheme in the malicious nodes, as may be expected in a hostile environment has not been investigated. Trust and energy aware routing protocol was proposed for WSNs and it aims to report the energy limitations. By keeping resource constrained distinctive of WSNs in mind, the design of Trust and energy aware routing protocol is centered on trustworthiness and energy efficiency. Routing Protocol is proficient of energetically sensing and segregate misbehave nodes during trust assessment phase whereas energy awareness feature was amalgamated in route setup phase which aids in improved load balancing among trust nodes [15].

The hierarchical trust management for WSNs performs multi path routing when intrusion detected in WSNs. It assesses the trustworthiness of node utilizing subjective 
trust (performance at running time) and objective trust (node status) [16]. Higher energy consumption for the duration of data gathering and delivery process is decreased utilizing a clustering method; hierarchical routing exploits upon these advantages by the division of nodes into clusters. Clustering method generally selects the node with more energy as Cluster Head $(\mathrm{CH})$, which collects the information and transmitted through lower-energy nodes by clustering method [17]. The Cluster based routing protocols (CBR-MOBILE) is proposed to face the challenges of packet loss and energy consumption in Hybrid networks like some sensor nodes are fixed, and other are mobile. It is traffic adaptive protocol that assigns timeslots of mobile nodes which are moves out of cluster can be reassign to the incoming mobile nodes into that cluster. Based on receiving signal strength, data is transmitted to the cluster head [26].

A secure mobile data collector is introduced in clusters to collect the data from cluster head, and forward to the base station. Authors proposed and analyzed three protocols for secure data collection, and it follows tree based connection management among sensor nodes [27]. Optimization of mobile data collector speed, and it's mobility is changed adaptively based on the requirements of network, are the objectives of paper, and this can be achieved by providing cooperation between sensing nodes, and mobile data collector nodes [28].

The comparison of clustering protocols regarding different attributes was represented in Table 1. In this paper, trust based routing protocol, sub-clustering algorithm was proposed to decrease energy consumption, and leads to a rise in network lifetime, network throughput were assessed. In WSNs, trust stipulates the dependability or trustworthiness of sensor node. The trust value was calculated based on the actual sensed data of the sensors in WSNs is termed as data trust.

Table 1. Comparison of clustering protocols with clustering attributes

\begin{tabular}{|l|l|l|l|l|l|l|}
\hline $\begin{array}{c}\text { Clustering } \\
\text { Protocol }\end{array}$ & \multicolumn{1}{|c|}{$\begin{array}{c}\text { Clustering } \\
\text { Approach }\end{array}$} & $\begin{array}{c}\text { Clustering } \\
\text { Method }\end{array}$ & $\begin{array}{c}\text { Number of } \\
\text { Cluster head }\end{array}$ & Network type & $\begin{array}{c}\text { Energy } \\
\text { Model }\end{array}$ & $\begin{array}{c}\text { Connectivity } \\
\text { to the BS }\end{array}$ \\
\hline EEHC[18] & Prob./Energy & Distributed & Variable & $\begin{array}{l}\text { Homogeneous/ } \\
\text { Heterogeneous }\end{array}$ & First Order & One-hop \\
\hline LEACH [19] & Prob./Randm & Distributed & Variable & Homogeneous & First Order & One-hop \\
\hline S-EEP [20] & Prob./Energy & Distributed & Variable & Homogeneous & First Order & One-hop \\
\hline $\begin{array}{l}\text { TPSO-CR } \\
{[21]}\end{array}$ & PSO & Centralized & Fixed & $\begin{array}{l}\text { Homogeneous/ } \\
\text { Heterogeneous }\end{array}$ & $\begin{array}{l}\text { Discrete } \\
\text { (CC2420) }\end{array}$ & Multi-hop \\
\hline M-EEP[20] & Greedy & Centralized & Variable & $\begin{array}{l}\text { Homogeneous/ } \\
\text { Heterogeneous }\end{array}$ & First Order & $\begin{array}{l}\text { One-hop/ } \\
\text { Multi-hop }\end{array}$ \\
\hline $\begin{array}{l}\text { LEACH-C } \\
{[19]}\end{array}$ & SA & Centralized & Fixed & Homogeneous & First Order & One-hop \\
\hline
\end{tabular}

\section{Methodology}

In wireless sensor networks, there are a number of trust based routing protocols was proposed. To enhance the network lifetime, efficiency, throughput, various techniques are implemented based on trust values of nodes. In existing works, mobile nodes $(\mathrm{MN})$ are introduced in fixed wireless sensor networks to gather the infor- 
mation from sensor nodes and send it to $\mathrm{CH}$. This type of implementations gives great results in military applications, plateaus, hill and valley places, etc. In this research, trust based routing protocol and sub-clustering algorithm was proposed in WSN. Fig. 1 displays the scenario of trust node in WSN. Cluster node is divided into the sub clusters and we assign some sub-cluster nodes as trust nodes. In sub cluster the mobile nodes are moving around the sensor node and $\mathrm{MN}$ comes near to the $\mathrm{SN}$ then node awake and sends data to MN. If the mobile node is stationary, the trust node will send the data to $\mathrm{MN}$.
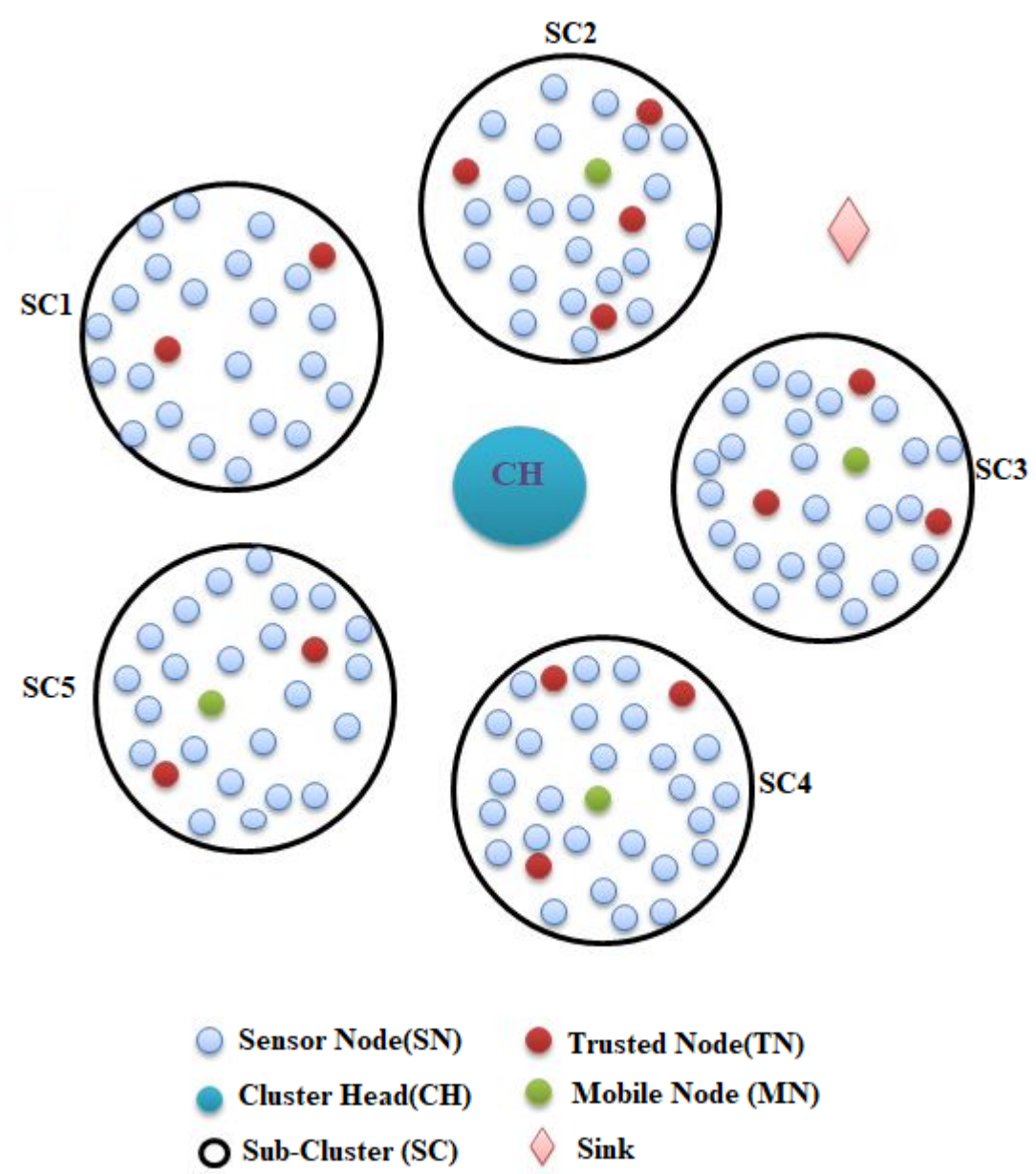

Fig. 1. Scenario of trusted node in WSN

\subsection{Proposed energy efficient trust node based routing protocol}

Design flow of trust node in WSN: The data flow diagram of proposed EETRP protocol and sub clustering algorithm was given in Fig. 2 which represents the for- 
mation of the sub cluster region and energy harvesting was described by the below important steps.

Step1: The BS monitors WSNs sensor nodes with cluster head and it forms cluster into sub cluster region separately.

Step2: Deployment of sensor nodes (SN) selected randomly. In the sensor network, all sensor nodes are arbitrarily deployed in a circular area with a radius of $\mathrm{R}$. The network model can be clarified as an undirected connectivity graph $G(S, E)$, where $S$ is the set of all SN and E $(i, j)$ is the set of wireless link amongst node $i$ and node $\mathrm{j}$.All the $\mathrm{SN}$ are homogeneous and stationary [22]. We divide the entire sub cluster region into several sectors named as fixed sensor nodes, one mobile node and two or three trust nodes.

Step3: Deployment of one mobile node (MN) for each sun-cluster.

Step4: Move the mobile node $(\mathrm{MN})$ for first round (odd number of round) around the Sub-cluster (SC).

Step5: When the mobile node $(\mathrm{MN})$ comes near to the sensor node then sensor node (SN) awake from sleep mode and sends the data to the mobile node (MN) (same procedure for every node).

In WSNs, the life cycle can be influenced by the residual energy of each node. Thus, energy plays an important role in the construction and operation of the network. All the sub cluster nodes have restricted energy, which will be consumed when the nodes send or receive data [15]. The first-order radio model is used as the energy consumption model. The quantity of energy consumption in transmitting $l$-bit packet from node $i$ to node $j$ can be represented by:

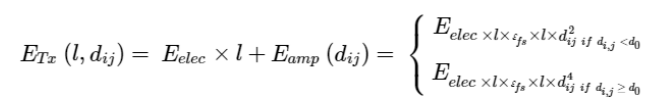

Here $E_{\text {elec }}$ is the energy necessary for driving and controlling electronic components. $E_{a m p}$ Depends on the Radio Frequency (RF) amplifier's consumed energy for free space $\varepsilon_{f s}$ and multipath fading channel models $\varepsilon_{m p}$ and $d_{i j}$ represents the Euclidean distance between node $i$ and node $j$. The threshold, $d_{0}$ is calculated as follows

$$
d_{0}=\sqrt{\frac{\varepsilon_{f s}}{\varepsilon_{m p}}}
$$

The node consumes the following amount of energy in receiving $E_{R x} l$-bit packet

$$
E_{R x}(l)=E_{\text {elec }} \times l
$$

Step 6: The mobile node (MN) will be stationary for second round (even number of rounds) then trust node (TN) sends data to the mobile node (MN) from their location. After sending the data SN will be stationary. 
A trust node of route will be calculated so as to ignore the encountering malicious nodes through the data-forwarding phase [23]. Trust nodes are initialized to 0 by default but since the model is totally local, nodes are free to initiate some trust node. The evolution of the trust node $n_{i}$ on the node $n_{j}$ is given by the following formula

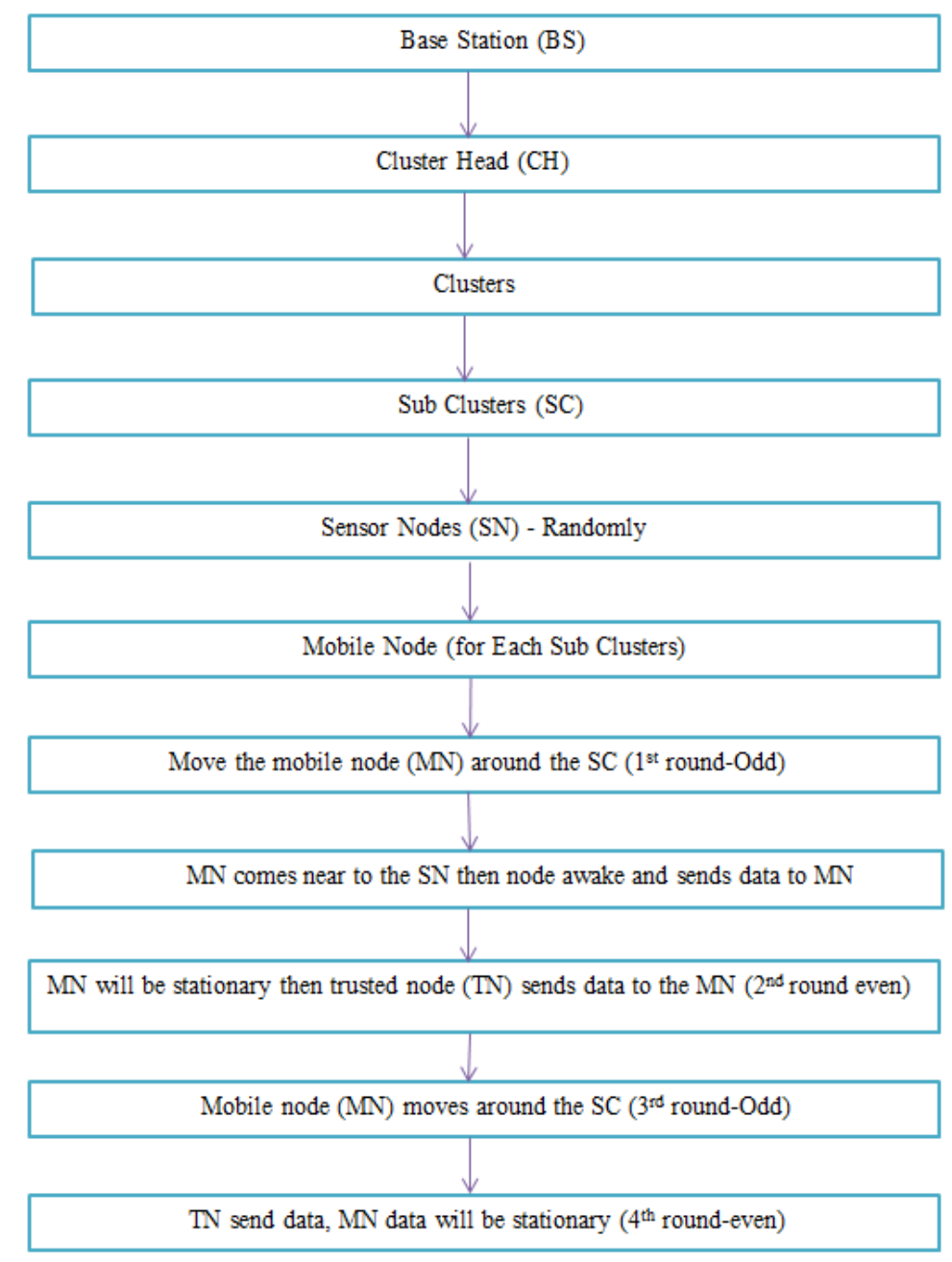

Fig. 2. Design flow of EETRP in WSN

$$
C_{n_{i}, n_{j}}^{D}(t)=\left\{\begin{array}{c}
-1 \quad \text { if } P_{n i, n j}(t)<0 \\
1-\propto^{P_{n i, n j}(t)} \quad \text { Otherwise }
\end{array}\right.
$$


Here $\propto \in(0,1)$, (higher is $\propto$, slower a direct trust goes from 0 to 1 with time, vice versa) and $P_{n i, n j}(t)$ depends on the number of positive experiences $P_{n i, n j}^{+}(t)$ (the number of good behaviors) and the number of negative experiences $P_{n i, n j}^{-}(t)$ (the number of bad behaviors) of $n_{i}$ observed by $n_{i}$ until time $t$. The value of $P_{n i, n j}(t)$ is d number of bad behaviors) of $n_{j}$ observed by $n_{i}$ un

is defined as

$$
P_{n i, n j}(t)=P_{n i, n j}^{+}(t)-\beta * P_{n i, n j}
$$

Where $\beta(\beta \geqslant 1)$, is a parameter which allows ${ }_{\mathrm{u}}$, $\beta$, the modulation of the importance of negative experiences (greater is $\beta$, larger is the influence of negative experiments), $\beta$ is introduced so that a definite number of faults may be tolerated. Both $\propto$ and $\beta$ should be relatively high to keep the efficacy of the model. According to the above formula,

- If a node always behaves well, its trust value will rapidly increase to 1

- If a node is moderately malicious or failing, its trust node will be stable

- If the node is malicious or quite failing, then it will immediately become untrust.

Step7: For third round (odd number of round) again mobile node (MN) moves around the sub cluster (SC)

Step8: In fourth round (even number of round) again trust node (TN) send data and the $\mathrm{MN}$ will be stationary.

Pseudo code for EETRP with sub-clustering algorithm: Event detection can be possible by checking the sensed data of trusted node with neighbor nodes data, to decide the event occurrence. Trusted nodes will send data to mobile node to avoid malfunctions or malicious attacks.

\section{Sub clustering algorithm}

No. of nodes $(n)=51$

Divide the clusters $(\mathrm{C})=$ node position

Calculate residual energy for each node.

For $(\mathrm{i}=1$ to $\mathrm{n})$

Dn $\leftarrow$ calculate degree difference $(\mathrm{n})$

$\mathrm{N}(\mathrm{n}) \leftarrow$ identify neighbors $(\mathrm{n})$

$\mathrm{Tc} \leftarrow$ calculate cumulative time (n)

$\mathrm{Cn} \leftarrow$ calculate the character $(\mathrm{n})$

$\mathrm{Wn} \leftarrow$ calculate the weight (n)

En $\leftarrow$ calculate the energy $(n)$

Call the combined weight based energy calculation

For (every $\mathrm{n} € \mathrm{~N}$ )

If $\mathrm{n}$ is high weight then

$\mathrm{CH} \leftarrow$ high weight $(\mathrm{n})$

Update in neighbor table

Else 
$\mathrm{CM} \leftarrow$ cluster member $(\mathrm{N})$

End if

End for

Cluster $\rightarrow$ division to $\mathrm{SCH}$

$\mathrm{SCH} \leftarrow$ Node position

Each $\mathrm{SCH} \leftarrow 1 \mathrm{MN}$

No of $\mathrm{MN}=$ validate the no. of clusters

\section{Energy Efficient Trust node based Routing Protocol}

No of rounds $=\mathrm{N}$

$\mathrm{N}=[\mathrm{n}+1 / 2],[2 \mathrm{n}+1 / 2]$

$\mathrm{N}=\sum_{n=0}^{N}[\mathrm{n}+1 / 2]$ for all odd rounds

$\mathrm{N}=\sum_{n=0}^{N}[2 \mathrm{n}+1 / 2]$ for all even rounds

When

$\mathrm{N}=$ odd round

$\mathrm{MN} \leftarrow$ data broadcast from $\mathrm{SCH}$

$\mathrm{N}=$ even round

$\mathrm{MN} \leftarrow$ data broadcast from trust nodes

Continuous sensing, and data collection in plateaus, military areas results large energy consumption, and effected with a limited lifetime due to their uneven placement. Hence, this protocol proposes, an alternative round of data collection in WSN to improve lifetime of the network. During initial round, Mobile data collector will collect the data from sensed nodes individually and aggregated, forwarded the data to cluster head. Due to uneven placement of sensor nodes in plateaus, they are not efficiently clustered as traditional approaches like tree structured clustering. Hence we collect data individually from each sensor nodes; due to this secure data, collection is possible, without any additional overhead. After completion of first round, Mobile data collector collects data from only trusted nodes, trusted nodes will check their sensed data with neighbors and forward to the nearest Mobile data collector. After data collection from trusted nodes, again the mobile data collector node will collect data from sensor nodes individually in next round of data collection, to avoid failures in event detection, and malicious attacks. This alternative round of data collection gives lower values in energy consumption and higher values in Network lifetime. Based on application, we can increase the number of trusted node data collection rounds rather than mobile data collector rounds. By increasing the number of trusted nodes, throughput can be increased, and by increasing number of trusted nodes data collection rounds per cycle, throughput can be decreased. 


\section{Simulation Parameters}

This paper assumes 51 (i.e. $\mathrm{n}=51$ ) mobile sensor nodes that are arbitrarily deployed inside the area of $1320 * 1032$. The 3 kinds of nodes which include sink node, cluster node and trust nodes with the initial energy of $500 \mathrm{~J}$ respectively. The BS was located at any random place outdoor the sensing field. All the residual parameters are presented in Table 2. The SNs sense the data at a fixed rate and these SNs have always some data to send.

Table 2. Simulation Parameters

\begin{tabular}{|l|c|}
\hline \multicolumn{1}{|c|}{ Parameters } & Values \\
\hline Simulation Period & $100 \mathrm{~ms}$ \\
\hline Coverage Area & $1320^{*} 1032$ \\
\hline No of Nodes & 51 \\
\hline No of sink node & 1 \\
\hline No of mobile node & 5 \\
\hline No of Sub cluster & 5 \\
\hline No of Cluster Head & 1 \\
\hline Traffic Type & CBR \\
\hline Agent Type & UDP \\
\hline Routing protocol & EETRP \\
\hline Initial power & $100 \mathrm{~J}$ \\
\hline Transmission Power & $0.2 \mathrm{~J}$ \\
\hline Receiving Power & $0.2 \mathrm{~J}$ \\
\hline Queue Type & Drop-Tail \\
\hline
\end{tabular}

\section{$5 \quad$ Results and Discussion}

The results of proposed EETRP protocol with sub clustering algorithm has evaluated and compared the results with existed SPT, MLPA and GLBD by using ns 2 simulator. The simulation results has given the parameters of Network throughput, Network lifetime, Network energy efficiency, Network energy consumption, Control overhead, Network packet delivery ratio and Network packet loss. The simulation has been performed by using EETRP protocol with sub clustering algorithm and free space propagation. In WSN scenario, a SNs network contains total number of nodes (51) is installed arbitrarily above an area of size $1320 * 1032$ within the network field. Table 3 displays the evaluation of proposed EETRP protocol with sub clustering algorithm with existing trust algorithm in WSNs. 
Table 3. Comparison of existing algorithm with proposed TBRP Algorithm

\begin{tabular}{|c|c|c|c|c|}
\hline Algorithm & Aim & $\begin{array}{c}\text { Technique } \\
\text { used }\end{array}$ & $\begin{array}{c}\text { Energy } \\
\text { efficient }\end{array}$ & Goals achieved \\
\hline $\begin{array}{l}\text { Trust and Energy aware } \\
\text { routing protocol Wireless } \\
\text { Sensor Network }\end{array}$ & $\begin{array}{l}\text { Best path to reach } \\
\text { destination }\end{array}$ & $\begin{array}{l}\text { Trust, energy } \\
\text { Location calculation }\end{array}$ & Yes & $\begin{array}{l}\text { Avoid suspicious } \\
\text { node }\end{array}$ \\
\hline $\begin{array}{l}\text { Providing trust in wireless } \\
\text { sensor network using a } \\
\text { bio-inspired Technique. }\end{array}$ & $\begin{array}{l}\text { Trust and reputation, } \\
\text { Reputable path }\end{array}$ & $\begin{array}{l}\text { Ant colony } \\
\text { Optimization system }\end{array}$ & No & $\begin{array}{l}\text { Avoid Misbe- } \\
\text { having } \\
\text { server }\end{array}$ \\
\hline $\begin{array}{l}\text { A Direct Trust Dependent } \\
\text { Link State Routing Proto- } \\
\text { col using Route Trust For } \\
\text { WSNs(DTLSRP) }\end{array}$ & Trustworthy route & Direct trust calculation & No & Routing attacks \\
\hline $\begin{array}{l}\text { Neighbor based malicious } \\
\text { node discovery in wireless } \\
\text { sensor networks }\end{array}$ & $\begin{array}{l}\text { Precise malicious } \\
\text { node detection }\end{array}$ & $\begin{array}{l}\text { Data smoothing variation } \\
\text { test and confidence level } \\
\text { evaluation }\end{array}$ & No & $\begin{array}{l}\text { Low false alarm } \\
\text { rates. }\end{array}$ \\
\hline $\begin{array}{l}\text { TARF: A trust aware } \\
\text { routing framework for } \\
\text { wireless sensor networks }\end{array}$ & $\begin{array}{l}\text { Routing includes } \\
\text { Trustworthiness of } \\
\text { node }\end{array}$ & $\begin{array}{l}\text { Trust manager, energy } \\
\text { watcher }\end{array}$ & Yes & $\begin{array}{l}\text { Authentication, } \\
\text { replay of routing } \\
\text { information }\end{array}$ \\
\hline Proposed: EETRP protocol & $\begin{array}{l}\text { Reducing Energy } \\
\text { consumption and } \\
\text { Prolong network life } \\
\text { time }\end{array}$ & $\begin{array}{l}\text { Trust based routing proto- } \\
\text { col with sub clustering } \\
\text { algorithm }\end{array}$ & Yes & $\begin{array}{l}\text { Avoid malicious } \\
\text { node and trust- } \\
\text { worthy }\end{array}$ \\
\hline
\end{tabular}

\subsection{Network throughput}

The amount of packets received by the BS from non-base station nodes is called throughput. As number of nodes upsurges throughput will increase in all other data aggregation and EETRP protocol with sub clustering algorithm data aggregation provides throughput as 1153 Mbps. The proposed EETRP Protocol with sub clustering algorithm provides maximum throughput when compared to existing GLBD [24], MLPA and SPT [10] algorithm. Fig. 3 defines the throughput evaluation amongst EETRP Protocol with sub clustering algorithm and its contestant's. Throughput of EETRP protocol with sub clustering algorithm was found to be $1153 \mathrm{Mbps}$ which is greater than SPT (886), MLPA (542) and GLBD (244) algorithm respectively. Throughput depends on number of packets received for a specific node. By increasing number of trusted nodes, the throughput can increase, but by depending on more number of trusted data collection rounds, throughput will decreases. The throughput of proactive routing protocol is openly linked to cluster lifetime, higher cluster life time effects higher throughput. EETRP Protocol with sub clustering algorithm life time is superior to its opponents, then it has superior throughput that is evidently stated in Fig. 3. Due to mobile data collector node, data collected individually from all sensor nodes, therefore data faults, and malicious attacks are less compared with existed protocols. 


\subsection{Network lifetime}

From the beginning of the operation of the sensor network to the battery depletion of the last node is the network lifetime. In the simulation, the lifetime is assessed in terms of a cycle in WSN. Fig. 4 shows that EETRP protocol with sub clustering algorithm has higher stable lifetime than the other three hierarchical routing techniques. Due to maintaining large number of sensor nodes in sleep state until mobile data collector collects data from nodes individually, and by maintaining mobile data collector in idle condition in trust node based data collected rounds, the energy consumption is reduced greatly, and lifetime of the network also increases potentially than existing algorithms. The network lifetime of EETRP Protocol with sub clustering algorithm was found to be $68 \%$ which is greater than GLBD (56\%), MLPA (35\%) and SPT (20\%) algorithm respectively. Fig. 4 clearly represents the EETRP protocol with sub clustering algorithm was far superior and more efficient in expressions of the number of active nodes and the network lifetime.

\subsection{Network energy efficiency}

Network Energy and Network Lifetime are Key parameters of WSN. Those two parameters are linear in Relation. The Performance of WSN depends on Network Energy Efficiency. Energy efficiency upsurge the performance of WSNs, and it can be expressed as the ratio of networks lifetime and sensor node's energy. The Lifetime and Energy Efficiency of Network are enhanced when the sensor node's Energy is stable. Total energy consumed by all the nodes in the network is expressed by

$$
\text { Energy Efficiency }=\sum_{i=1}^{N} E_{i}
$$

$E_{i}$ Signifies the energy consumed by the nodes in rounds. It decreases the energy wastage and performs the number of rounds with limited energy. The network Energy efficiency of EETRP Protocol with sub clustering algorithm is $68 \%$ which is greater than GLBD, MLPA and SPT 56\%, 35\% and 20\% [10] algorithm respectively.

\subsection{Network packet delivery ratio}

The packet delivery ratio lies in the loss-rate explanation observed by transport protocols and disturbs the entire network throughput. This metric accentuates the correctness and completeness of the routing protocol. Fig. 5 show that the comparison of packet delivery ratio between proposed EETRP Protocol with existing algorithm.

Packet Delivery Ratio $=\frac{\text { Total number of packets that have been sent from the source node }}{\text { Total number of packet that had reached the destination }}$

The EETRP protocol with sub clustering algorithm shows a considerable improvement in packet delivery ratio over GLBD, MLPA and SPT. As the integer of nodes upsurges the packet delivery ratio will increase in all other data aggregation and EETRP Protocol with sub clustering algorithm data aggregation provides maximum 
packet delivery ratio for 51 nodes was $97.77 \%$, which is higher than $91.77 \%, 77 \%$ and $72 \%$ GLBD, MLPA [25] and SPT respectively.

\subsection{Network energy consumption}

Energy used by the network is proportional to the diameter of the network. Table 4 shows comparison of various simulated parameters. Fig. 6 depicts the energy consumption amongst proposed EETRP Protocol with sub clustering algorithm. Due to deployment of trusted nodes per each sub cluster, remaining sensor nodes can spend more time in sleep state, until mobile collector gives wake-up notification. On other hand mobile data collector can be placed idle in even number of rounds, and it can collect data directly from trusted nodes. Due to these reasons, network consumes very less power compared with conventional protocols. Energy consumption of EETRP Protocol with sub clustering algorithm was found to be $32 \%$ which is lesser than GLBD (44\%), MLPA (65\%) [25] and SPT (80\%) respectively.

Table 4. Comparison of various simulated parameters

\begin{tabular}{|l|c|c|c|c|}
\hline \multicolumn{1}{|c|}{ Parameters } & SPT & MLPA & GLBD & EETRP \\
\hline Control Overhead & 1564 & 1265 & 969 & 2373 \\
\hline $\begin{array}{l}\text { Packet Delivery } \\
\text { Ratio (\%) }\end{array}$ & 72 & 77 & 91.77 & 97.77 \\
\hline Network Loss & 564 & 432 & 207 & 154 \\
\hline $\begin{array}{l}\text { Network Energy } \\
\text { Efficiency (\%) }\end{array}$ & 20 & 35 & 56 & 32 \\
\hline $\begin{array}{l}\text { Network Energy } \\
\text { Consumption (\%) }\end{array}$ & 80 & 65 & 886 & 1153 \\
\hline $\begin{array}{l}\text { Network Through- } \\
\text { put (Kbps) }\end{array}$ & 244 & 542 & 54 & 66 \\
\hline $\begin{array}{l}\text { Network Lifetime } \\
\text { \%) }\end{array}$ & 21 & 36 & & \\
\hline
\end{tabular}

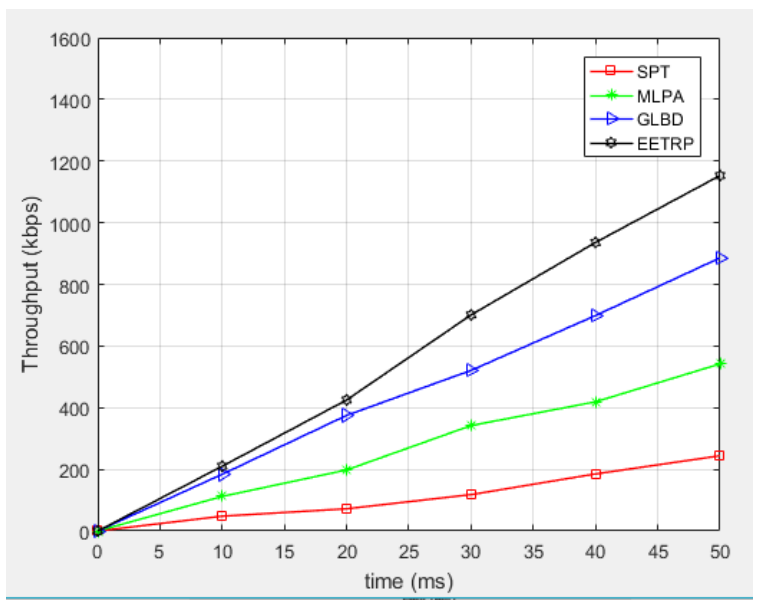

Fig. 3. Comparison of network throughput 


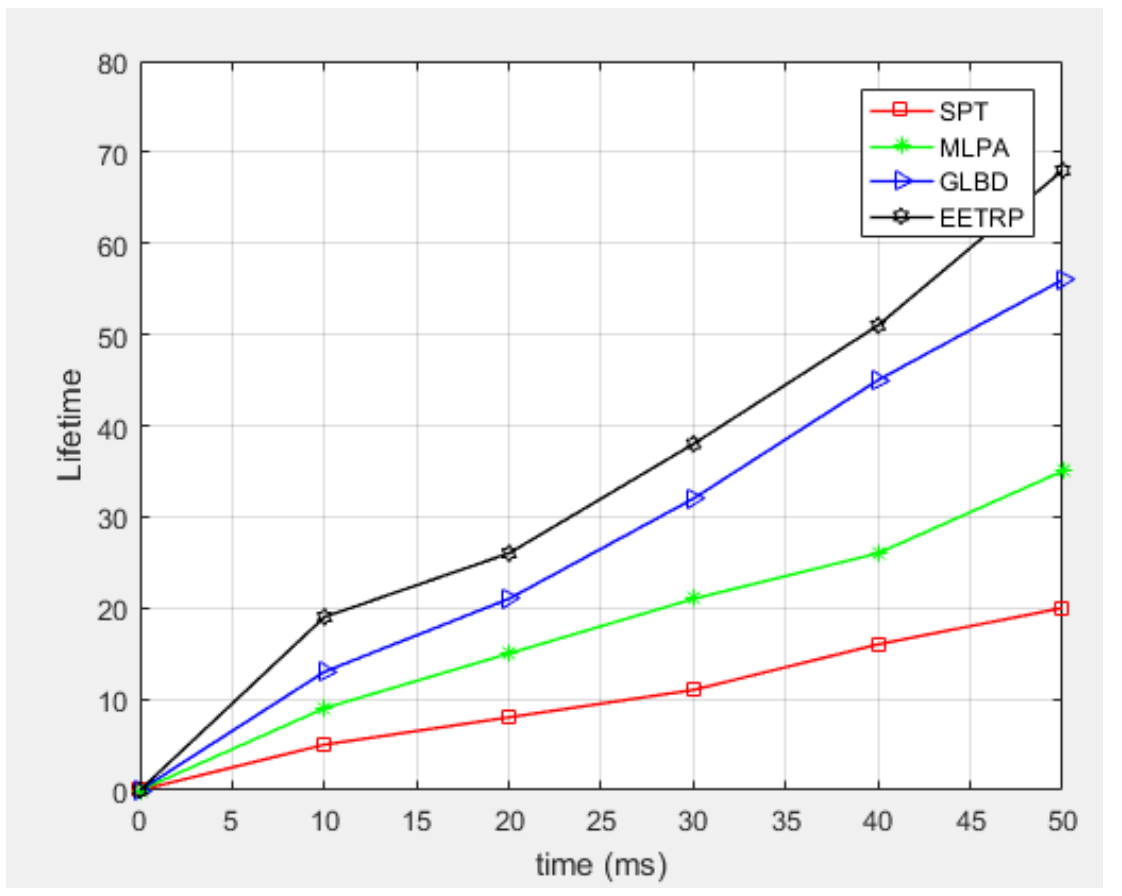

Fig. 4. Comparison of network lifetime

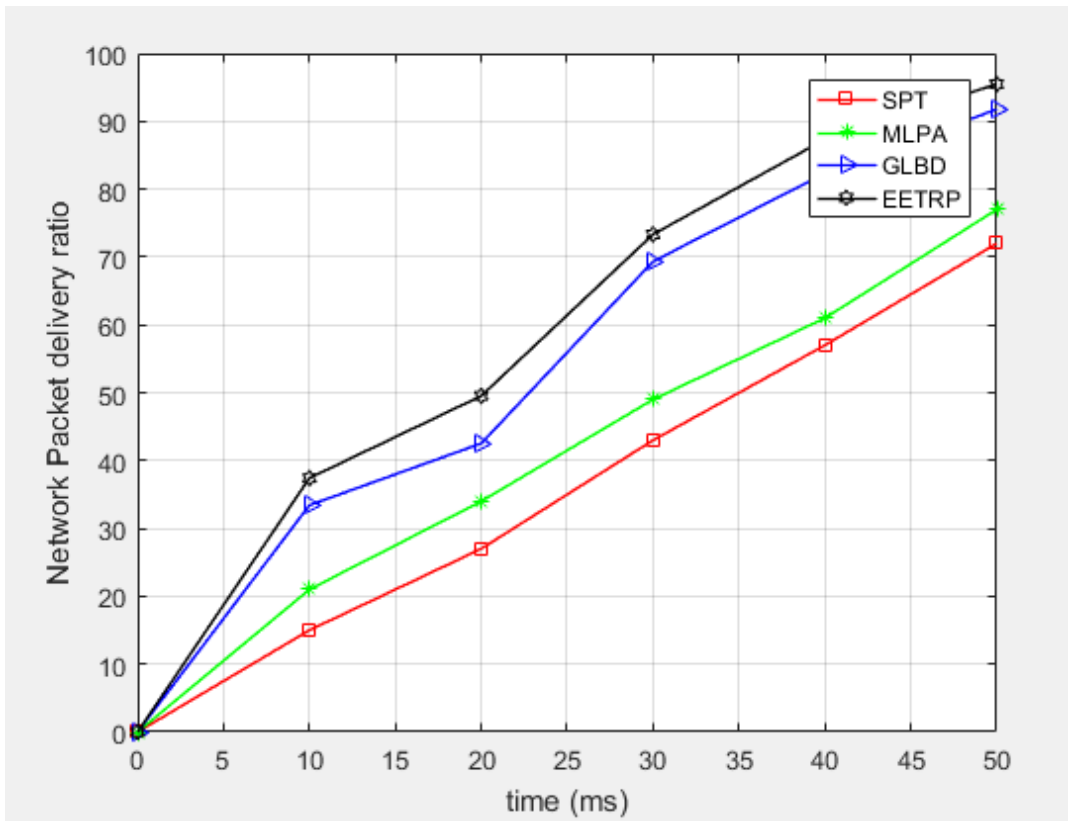

Fig. 5. Comparison of network packet delivery ratio 


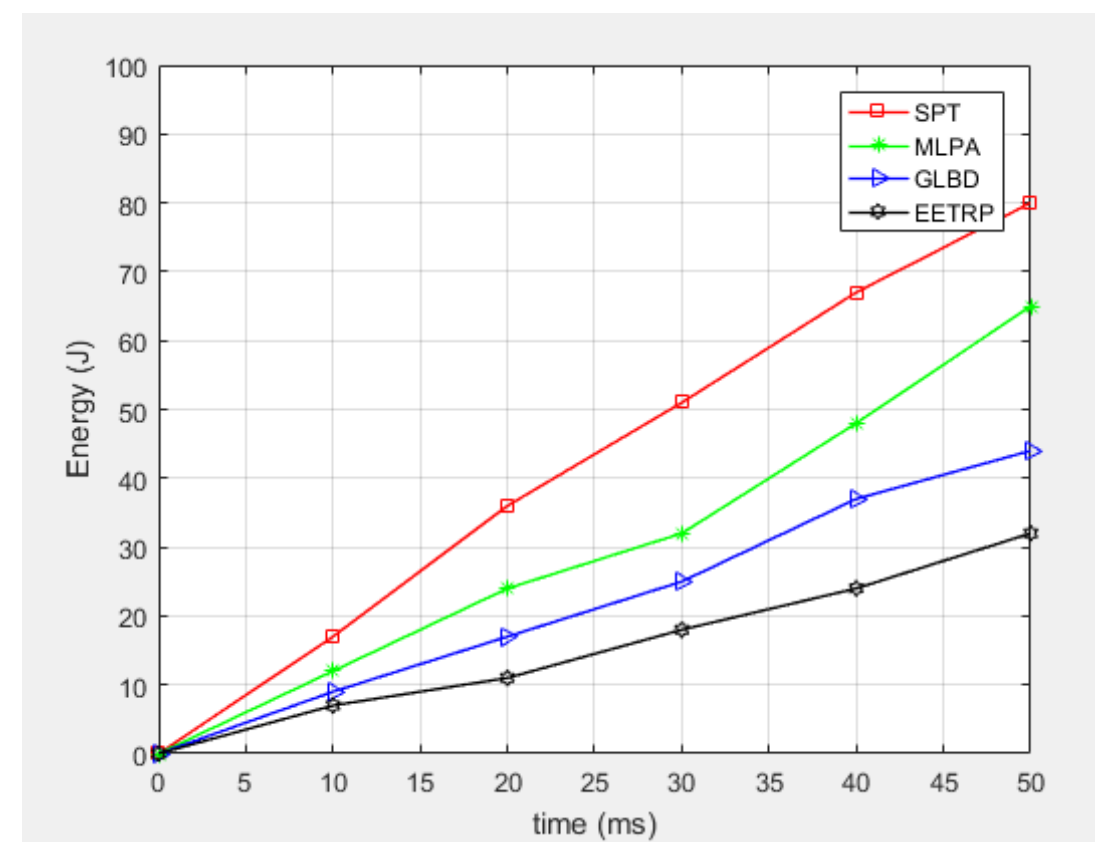

Fig. 6. Comparison of network energy consumption

\subsection{Parametric Analysis of Lifetime, Energy Consumption based on No of Trusted Routing iterations per Cycle.}

Parametric analysis can be obtained by varying the trusted routing iteration number with respect to mobile routing iteration number. By increasing trusted round Iteration number, the lifetime of the network increases linearly. On the other hand the network energy consumption is keeps on decreasing. The problem arises in this type of analysis is TRUST Dependency. When trust iterations are more in a cycle, then QOS may decrease.

\begin{tabular}{|l|c|c|c|c|}
\hline \multicolumn{1}{|c|}{ Parameter } & $\begin{array}{c}\text { Alternative rounds } \\
\text { (one trusted routing } \\
\text { iteration per onemo- } \\
\text { bile routing iteration) }\end{array}$ & $\begin{array}{c}\text { Two trusted } \\
\text { routing iterations } \\
\text { per one mobile } \\
\text { routing iteration }\end{array}$ & $\begin{array}{c}\text { Three trusted } \\
\text { routing } \\
\text { iterations per } \\
\text { one mobile } \\
\text { routing } \\
\text { iteration }\end{array}$ & $\begin{array}{c}\text { Four trusted } \\
\text { routing } \\
\text { iterations per } \\
\text { one mobile } \\
\text { routing } \\
\text { iterations }\end{array}$ \\
\hline $\begin{array}{l}\text { Network energy } \\
\text { consumption }\end{array}$ & 32 & 30 & 26 & 22 \\
\hline Network lifetime & 68 & 74 & 81 & 89 \\
\hline
\end{tabular}




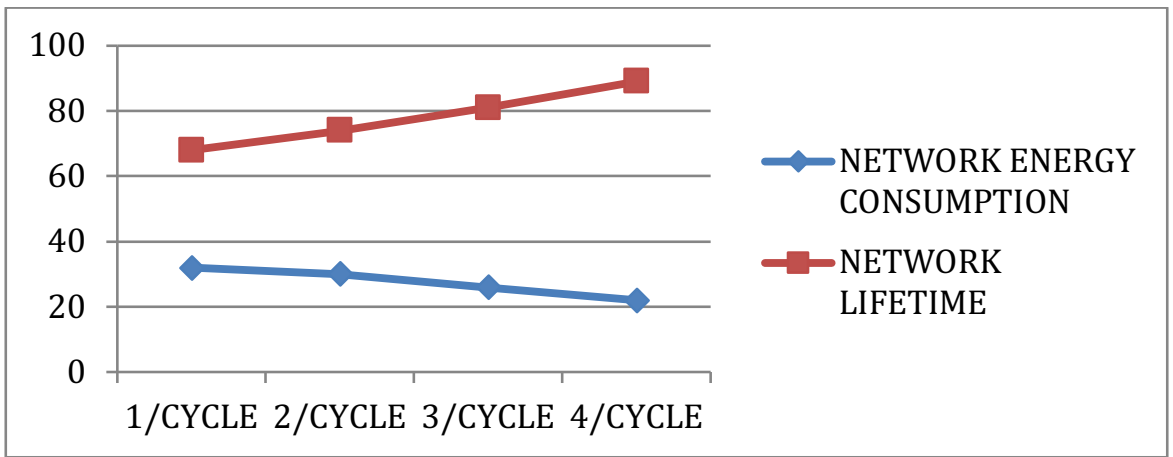

The graph shows variations of energy consumption and lifetime of the network for various trusted iterations.

\section{Conclusion}

The sensor nodes in WSNs have limited computation, storage and interaction abilities. In this paper, EETRP protocol with sub clustering algorithm in WSNs is presented to balance energy and prolong network lifetime. This algorithm divides the clusters into several sub clusters and assigned each sub cluster with a mobile node. Sensor nodes perform the data interaction by the sub clustering structure and trust nodes are depends on data collection, thus increasing the energy efficiency of network. Therefore, the proposed approach improves the energy efficiency and network lifetime at the same time. The simulation outcomes indicate that energy consumption of network is greatly reduced by our proposed energy efficient trust based routing algorithm while compared with other clustering algorithm.

\section{$7 \quad$ References}

[1] Baroudi, U., Al-Roubaiey, A., Mekid, S., Bouhraoua, A. (2014). Delay characterization and performance evaluation of cluster-based WSN with different deployment distributions. Future Generation Computer Systems, 39, 100-110. https://doi.org/10.1 016/j.future.2014.02.011

[2] Younis, O., Krunz, M., Ramasubramanian, S. (2006). Node clustering in wireless sensor networks: recent developments and deployment challenges. IEEE network, 20, 3, 20-25. https://doi.org/10.1109/MNET.2006.1637928

[3] Amin, R., Islam, S. H., Biswas, G. P., Obaidat, M. S. (2018). A robust mutual authentication protocol for WSN with multiple base-stations. Ad Hoc Networks, 75, 1-18. https://doi.org/10.1016/j.adhoc.2018.03.007

[4] Gambhir, A., Payal, A., Arya, R. (2018). Performance analysis of artificial bee colony optimization based clustering protocol in various scenarios of WSN. Procedia Computer Science, 132, 183-188 https://doi.org/10.1016/j.procs.2018.05.184

[5] Mahajan, S., Malhotra, J., \& Sharma, S. (2014). An energy balanced QoS based cluster head selection strategy for WSN. Egyptian Informatics Journal, 15, 3, 189-199. https://doi.org/10.1016/j.eij.2014.09.001 
[6] Adnan, M. A., Razzaque, M. A., Abedin, M. A., Reza, S. S., Hussein, M. R. (2016). A novel cuckoo search based clustering algorithm for wireless sensor networks. In Advanced Computer and Communication Engineering Technology, 621-634. https://doi.org/10.1 007/978-3-319-24584-3 53

[7] Huaizhi Li and Mukesh Singhal, (2007). Trust Management in distributed Systems. IEEE Computer Society, 45-53. https://doi.org/10.1109/MC.2007.76

[8] Howard, A., Mataric, M. J., Sukhatme, G. S. (2002). An incremental self-deployment algorithm for mobile sensor networks. Autonomous Robots, 13, 2, 113-126. https://doi.org/10.1023/A:1019625207705

[9] Wei, D., Jin, Y., Vural, S., Moessner, K., Tafazolli, R. (2011). An energy-efficient clustering solution for wireless sensor networks. IEEE transactions on wireless communications, 10, 11, 3973-3983. https://doi.org/10.1109/TWC.2011.092011.110717

[10] Shan, M., Chen, G., Luo, D., Zhu, X., Wu, X. (2014). Building maximum lifetime shortest path data aggregation trees in wireless sensor networks. ACM Transactions on Sensor Networks (TOSN), 11, 1, 11. https://doi.org/10.1145/2629662

[11] Younis, O., Fahmy, S. (2004). HEED: a hybrid, energy-efficient, distributed clustering approach for ad hoc sensor networks. IEEE Transactions on mobile computing, 3, 4, 366379. https://doi.org/10.1109/TMC.2004.41

[12] Liu, Z., Zheng, Q., Xue, L., Guan, X. (2012). A distributed energy-efficient clustering algorithm with improved coverage in wireless sensor networks. Future Generation Computer Systems, 28, 5, 780-790. https://doi.org/10.1016/j.future.2011.04.019

[13] Han, X., Cao, X., Lloyd, E. L., Shen, C. C. (2010). Fault-tolerant relay node placement in heterogeneous wireless sensor networks. IEEE Transactions on Mobile Computing, 9,5, 643-656. https://doi.org/10.1109/TMC.2009.161

[14] Chuanhe, H., Yong, C., Wenming, S., Hao, Z. (2007). A trusted routing protocol for wireless mobile ad hoc networks, IET Conference on Wireless, Mobile and Sensor Networks (CCWMSN 2007), 406 - 409.

[15] Ahmed, A., Bakar, K. A., Channa, M. I., Haseeb, K., Khan, A. W. (2016). A trust aware routing protocol for energy constrained wireless sensor network. Telecommunication Systems, 61, 1, 123-140. https://doi.org/10.1007/s11235-015-0068-8

[16] Bao, F., Chen, R., Chang, M., Cho, J. H. (2012). Hierarchical trust management for wireless sensor networks and its applications to trust-based routing and intrusion detection. IEEE transactions on network and service management, 9, 2, 169-183. https://doi.org/10.1109/TCOMM.2012.031912.110179

[17] Muthukumaran, K., Chitra, K., \& Selvakumar, C.(2017). An energy efficient clustering scheme using multilevel routing for wireless sensor network. Computers \& Electrical Engineering, 69, 642-652.

[18] Kumar, D., Aseri, T. C., Patel, R. B. (2009). EEHC: Energy efficient heterogeneous clustered scheme for wireless sensor networks. Computer communications, 32, 4, 662-667 https://doi.org/10.1016/j.comcom.2008.11.025

[19] Heinzelman, W. B., Chandrakasan, A. P., Balakrishnan, H. (2002). An application-specific protocol architecture for wireless microsensor networks. IEEE Transactions on wireless communications, 1(4): 660-670. https://doi.org/10.1109/TWC.2002.804190

[20] Kumar, D. (2013). Performance analysis of energy efficient clustering protocols for maximising lifetime of wireless sensor networks. IET Wireless Sensor Systems, 4, 1, 9-16. https://doi.org/10.1049/iet-wss.2012.0150

[21] Elhabyan, R. S., Yagoub, M. C. (2015). Two-tier particle swarm optimization protocol for clustering and routing in wireless sensor network. Journal of Network and Computer Applications, 52, 116-128. https://doi.org/10.1016/j.jnca.2015.02.004 
[22] Wang, J., Cao, Y., Li, B., Kim, H. J., Lee, S. (2017). Particle swarm optimization based clustering algorithm with mobile sink for WSNs. Future Generation Computer Systems, 76, 452-457. https://doi.org/10.1016/j.future.2016.08.004

[23] Xue, G. R., Lin, C., Yang, Q., Xi, W., Zeng, H. J., Yu, Y., Chen, Z. (2005). Scalable collaborative filtering using cluster-based smoothing. In Proceedings of the 28th annual international ACM SIGIR conference on Research and development in information retrieval, ACM. 114-121 https://doi.org/10.1145/1076034.1076056

[24] Tzung-Shi Chen, Hua-Wen Tsai and Chih-Ping Chu, "Gathering-Load-Balanced Tree Protocol for Wireless Sensor Networks," IEEE International Conference on Sensor Networks, Ubiquitous, and Trustworthy Computing (SUTC'06), Taichung, 2006, 8-13. https://doi.org/10.1109/SUTC.2006.67

[25] Cheng, S. T., Wu, M. (2009). Optimization of multilevel power adjustment in wireless sensor networks. Telecommunication Systems, 42, 1, 109-121. https://doi.org/10.1 007/s11235-009-9173-x

[26] Awwad, Samer A. B. and Ng, Chee Kyun and Noordin, Nor Kamariah and A. Rasid, Mohd Fadlee (2011) Cluster based routing protocol for mobile nodes in wireless sensor network. Wireless Personal Communications, 61, 2, 251-281. https://doi.org/10.1007/s11277-010-0022-8

[27] Poornima, A.S. \& Amberker, B.B. (2011). Secure data collection using mobile data collector in clustered wireless sensor networks. IET Wireless Sensor Systems, 1, 85 - 95 https://doi.org/10.1049/iet-wss.2010.0086

[28] Sayyed, Ali \& Buss Becker, Leandro. (2015). Optimizing speed of mobile data collector in Wireless Sensor Network. 1-6. International Conference on Emerging Technologies (ICET) https://doi.org/10.1109/ICET.2015.7389213

\section{Authors}

Nandoori Srikanth is one of the part time Ph.D. scholars in Koneru Lakshmiah Educational Foundation, from the department of Electronics \& Communication Engineering. He published many research papers in various reputed journals and he is working as an Assistant professor in NRI Institute of Technology. His Research area is Wireless Sensor Networks. His research interests are wireless communications, signal processing.

Muktyala Sivaganga Prasad is one of the Professors in Koneru Lakshmiah Educational Foundation, from the department of Electronics \& Communication Engineering. He published many research papers in various reputed International journals and he guided many more researchers in the fields of wireless communication, Antennas and wireless sensor networks. His research interests are wireless communications, Antennas and signal processing.

Article submitted 2019-01-19. Resubmitted 2019-03-01. Final acceptance 2019-03-02. Final version published as submitted by the authors. 